\title{
A case of autism showing clinical improvements after cellular Therapy along with PET CT evidence
}

\begin{abstract}
Autism is a complex neurodevelopmental disorder defined by a triad of deficits including impaired social interaction, communication and behaviour. With greater understanding of mechanism of action of cellular therapy it is now possible to address the pathology of autism. Here is a case of a six and a half year old boy with autism who was administered autologous bone marrow mononuclear cells (BMMNCs) intrathecally followed by an intensive rehabilitation program. On follow up at 3 months and 7 months post intervention, he showed significant symptomatic improvements with no major side effects. At the end of 7 months, ISAA score improved from 98 to 81. The Wee FIM showed improvement 80 to 89.1. CARS score reduced from 28.5 (mild to moderate autism) to 23.5 (mild autism). PET CT scan of the brain performed pre intervention and seven months post showed a balancing effect in the metabolism of affected areas. It also showed reduction in hypermetabolism of the frontal, parietal and temporal lobe bilaterally and increase in metabolism of the previously hypometabolic bilateral cerebelli. The changes observed on the PET CT scan of the brain correlated with clinical improvements. We hypothesize that cellular therapy holds great potential as a treatment modality for autism in combination with standard rehabilitation therapy. Randomized controlled trials are warranted to study their long term effects in treating autism.
\end{abstract}

Keywords: cellular therapy, pet ct scan, autism; stem cells, bone marrow mononuclear cells
Volume 2 Issue 4 - 2017

\author{
Alok Sharma,' Nandini Gokulchandran,' \\ Hemangi Sane, ${ }^{2}$ Pooja Kulkarni, ${ }^{2}$ Suhasini \\ Pai, $^{2}$ Vaishali Ganwir, ${ }^{3}$ Prerna Badhe \\ 'Department of Medical Services and Clinical Research, \\ NeuroGen Brain \& Spine Institute, India \\ ${ }^{2}$ Department of Research \& Development, NeuroGen Brain \& \\ Spine Institute, India \\ ${ }^{3}$ Department of Neurorehabilitation, NeuroGen Brain \& Spine \\ Institute, India
}

Correspondence: Pooja Kulkarni, Department of Research \& Development, NeuroGen Brain \& Spine Institute, Stem Asia Hospital and Research Centre, Sector - 40, Plot No. 19, Palm Beach Road, Seawoods (W), New Mumbai - 400706, India, Tel 919920200400, +912225283706, Email poojakul28@gmail.com

Received: February 10, 2017 | Published: April 17, 2017

\section{Introduction}

Autism is a neurodevelopmental disorder defined by a triad of deficits including impaired social interaction, communication and behavior. ${ }^{1}$ A complex association of genetic, environmental and biological risk factors are known to be responsible for causing autism however, its exact cause is unknown. Its neuropathology mainly involves defect in neural connectivity; neural migration; excitatoryinhibitory networks; abnormal dendritic morphology; neuroimmune disturbances; hypoperfusion; oxidative stress, calcium signaling, etc. ${ }^{2}$ Cellular therapy can address these pathologies by either replacing the lost or damaged neurons or by paracrine activity which may stimulate the endogenous cells to initiate repair.

Cellular therapy involves use of different types of cells which have the ability to repair and regenerate damaged tissues. ${ }^{3}$ In the past decade, researchers have extensively studied various aspects of cellular therapy in an array of disorders including neurological conditions which involves impairment of the central nervous system (CNS). These cells have unique ability to self renew, multiply and differentiate into any type of tissue making them viable for therapeutic and clinical applications, as the damaged CNS has a limited regenerative capacity. ${ }^{4}$ In this case report, we highlight the potential of cellular therapy as a therapeutic modality in autism.

We administered a diagnosed case of autism with autologous bone marrow mononuclear cells, intrathecally. These cells were selected for the study as they are easily obtainable, available in abundance and have no ethical issues. Autologous cells also do not involve any immune reaction which makes them a safe choice.

\section{Case representation}

We present the case of a 6 and $1 / 2$ year old boy diagnosed with autism, who underwent intrathecal administration of autologous BMMNCs. He had a birth history of preterm vaginal delivery without any neonatal complications such as kernicterus and seizures. At the age of 3years, his teacher noticed impaired social skills and poor eyecontact and was diagnosed as mild autism at the age of 31/2years based on clinical evaluation. There was no family history of autism. $\mathrm{He}$ achieved motor milestones at the right age but there was a delayed development in speech. Clinical manifestations included poor eye contact with presence of hyperactivity (sitting tolerance of only 5 minute) and restlessness. Emotional responses were inappropriate with presence of behavioral issues like throwing temper tantrums, putting things in mouth and crying without a reason. He also presented with poor social interaction, partial concentration deficit, irregular sleep patterns and could not convey his emotional needs. He had issues regarding food habits like not chewing properly, not eating fruits or soups. Functionally, he was dependent for all his Activities of Daily Living (ADLs) on his caretaker, with partial toilet training. He was on regular rehabilitation involving occupational and speech therapy since 1 year which did not show any improvements.

On the Indian Scale for Assessment of Autism (ISAA), his score was 98, while his Childhood Autism Rating Scale (CARS) and Functional Independence Measure (WeeFIM) scores were 28.5 (ASD) and 80 , respectively. MRI of the brain and EEG were normal. PET CT scan of the brain revealed hypometabolism in the bilateral cerebellar hemispheres and hypermetabolism in frontal, parietal and temporal lobes. 


\section{Materials and methods}

The protocol was reviewed and approved by Institutional Committee for Stem Cell Research and Therapy (IC-SCRT). The procedure of cellular therapy was explained in detail to the parents and a duly filled informed consent was obtained prior to the therapy.

Before the intervention, the patient underwent a complete neurological evaluation along with preoperative investigations to assess fitness for anesthesia. Granulocyte-Colony Stimulating Factor (G-CSF) (300 mcg) injections were administered subcutaneously, 72 hours and 24 hours prior to bone marrow aspiration. On the day of transplantation, approximately $100 \mathrm{ml}$ bone marrow was aspirated from the left anterior superior iliac spine under local anesthesia with sedation, using bone marrow aspiration needle and was collected in heparinized tubes. The BMMNCs were separated using density gradient method. Total numbers of mononuclear cells injected were 96X10 6 with 96\% viability. Fluorescence Activated Cell sorting (FACS) analysis showed CD34+ count to be 1619 cells/uL. These isolated cells were immediately injected intrathecally by a standard lumbar puncture procedure using an 18G Toughy needle between fourth and fifth lumbar vertebrae under local anesthesia with sedation. Simultaneously $20 \mathrm{mg} / \mathrm{kg}$ body weight methyl prednisolone in $500 \mathrm{~mL}$ Ringer Lactate was given intravenously to enhance survival of the injected cells. Patient was monitored for any adverse events.

\section{Results}

The patient was followed up regularly at 1 week, 3 months and 7 months. He showed significant improvements in this duration. Within a week of the intervention, his hyperactivity had reduced along with minimal improvement in eye contact. Sitting tolerance and attention span was improved. Speech improved significantly as he was able to make full sentences using rhyming words.

Three months after the intervention, there was remarkable reduction in hyperactivity and improvement in eye contact. He was able to convey his emotional needs to the parents. He tries to do things on his own and if he is unable to do so takes help from others without hesitation. A considerable reduction was observed in his repetitive behavior. He also started playing with toys and mingling with friends.

Seven months after the intervention, all the improvements were sustained. He was able to operate remote controlled cars and play with other kids. His social interaction further improved as he would try to initiate interaction. The habit of putting things in the mouth reduced. He could do all his ADLs independently and efficiently except toilet activities for which he was partially dependent on his mother for cleaning. He would attempt to eat by himself and also dress by himself. Awareness and judgement skills were better than before. Ability to learn new tasks, concept forming and problem solving also improved. He could independently solve puzzles. Sleep pattern had improved and he could sleep at a stretch of 8 hours.

On outcome measures, ISAA score showed a marked reduction from 98 to 81 (Table 1). CARS score reduced from 28.5 (mild to moderate autism) to 23.5 (mild autism). On the WeeFIM scale, his score improved from 80 to 89.1. On repeating the PET CT scan of the brain 7 months after intervention and comparing it to the previous scan, there was increased metabolism in the cerebellum and reduced metabolism in the frontal, parietal and temporal lobe bilaterally. These changes depicted overall improved metabolism towards normal (Figure $1 \&$ Table 2).
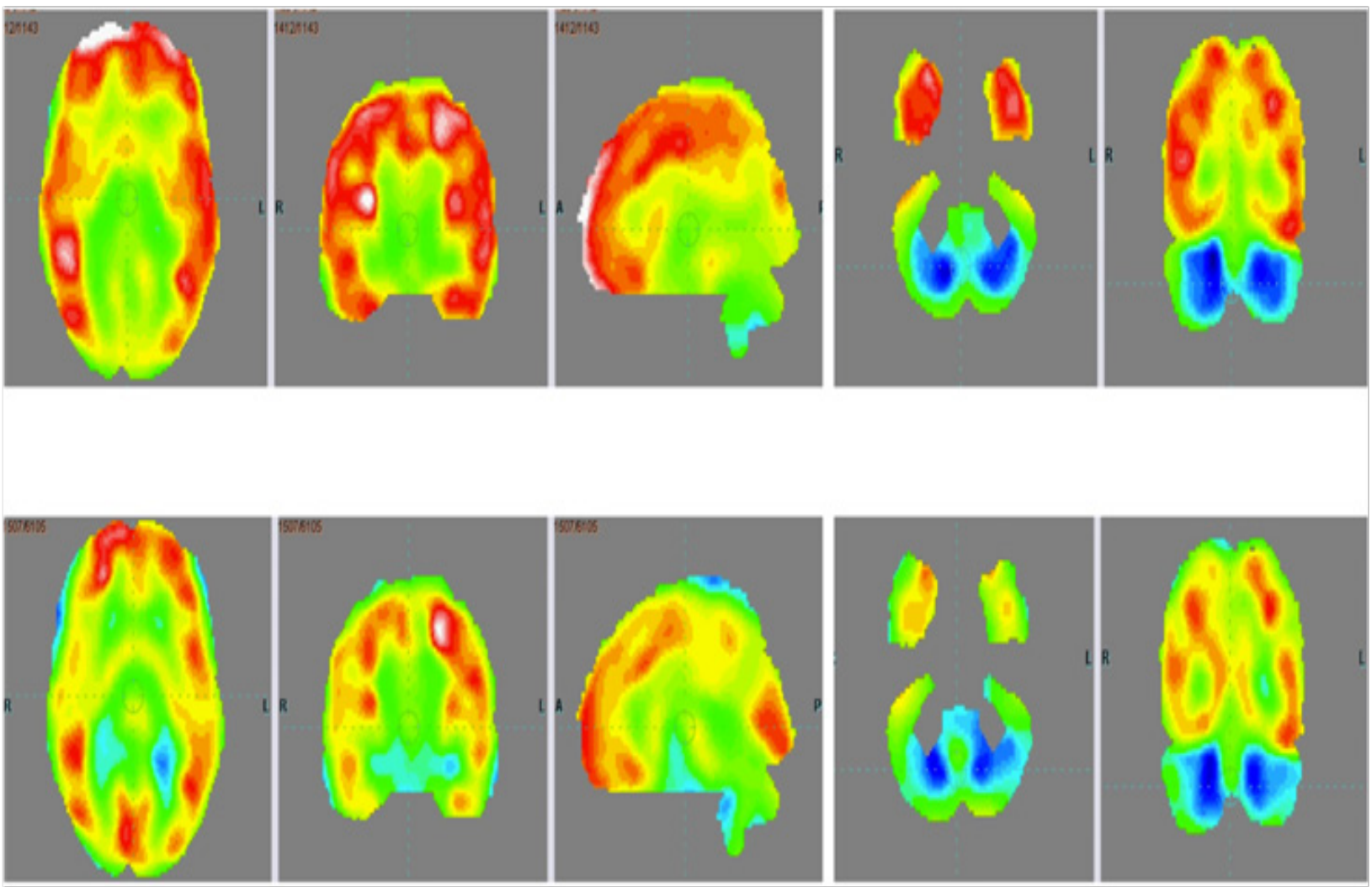

Figure I PET CT scan of the brain scan 7 months after first cellular transplantation showing reduction in the hypermetabolism in frontal lobe, parietal lobe and temporal lobe bilaterally and improved hypometabolism in bilateral cerebellar lobes as indicated by the circles. 
Table I Table giving details of the improvements in different sub components on ISAA at follow up of before and after first cellular transplantation

\begin{tabular}{|c|c|c|c|}
\hline $\begin{array}{l}\text { S. } \\
\text { no }\end{array}$ & Component & $\begin{array}{l}\text { Score before first cellular } \\
\text { transplantation }\end{array}$ & $\begin{array}{l}\text { Score post first cellular } \\
\text { transplantation }\end{array}$ \\
\hline A & Social relationship \& reciprocity & 27 & 20 \\
\hline I & Has poor eye contact & 2 & 1 \\
\hline 2 & Lacks social smile & 3 & 2 \\
\hline 3 & Remains aloof & 4 & 3 \\
\hline 4 & Does not reach out to others & 3 & 2 \\
\hline 5 & Unable to relate to people & 3 & 2 \\
\hline 6 & Unable to respond to social/environmental cues & I & I \\
\hline 7 & Engages in solitary and repetitive play activities & 4 & 4 \\
\hline 8 & Unable to take turns in social interaction & 5 & 4 \\
\hline B & Emotional responsiveness & 8 & 5 \\
\hline I & Shows inappropriate emotional response & 2 & 1 \\
\hline 2 & Shows exaggerated emotions & 2 & 1 \\
\hline 3 & Engages in self-stimulating emotions & I & I \\
\hline 4 & Lacks fear of danger & I & 1 \\
\hline 5 & Excited or agitated for no apparent reason & 2 & I \\
\hline C & Speech-language and communication & 27 & 24 \\
\hline $\mathrm{I}$ & Acquired speech and lost it & 4 & 4 \\
\hline 2 & $\begin{array}{l}\text { Has difficulty in using non-verbal language or gestures to } \\
\text { communicate }\end{array}$ & 4 & 4 \\
\hline 5 & Produces infantile squeals/unusual noises & 3 & 2 \\
\hline 6 & Unable to initiate or sustain conversation with others & 4 & 4 \\
\hline 7 & Uses jargon or meaningless words & 2 & 2 \\
\hline 8 & Uses pronoun reversals & 4 & 4 \\
\hline 9 & Unable to grasp pragmatics of communication & I & I \\
\hline D & Behavior patterns & 17 & 16 \\
\hline 1 & Engages in stereotyped and repetitive motor mechanisms & 2 & I \\
\hline 2 & Shows attachment to inanimate objects & 4 & 3 \\
\hline 3 & Shows hyperactivity/restlessness & 2 & 2 \\
\hline 4 & Exhibits aggressive behavior & 2 & 1 \\
\hline 5 & Throws temper tantrums & 2 & 2 \\
\hline 6 & Engages in self-injurious behavior & I & 4 \\
\hline 7 & Insists on sameness & 4 & 3 \\
\hline $\mathbf{E}$ & Sensory aspects & 7 & 6 \\
\hline 1 & Unusually sensitive to sensory stimuli & I & I \\
\hline 2 & Stares into space for long periods of time & I & $\mathrm{I}$ \\
\hline 3 & Has difficulty in tracking objects & I & I \\
\hline 4 & Has unusual vision & I & I \\
\hline
\end{tabular}


Table Continued.

\begin{tabular}{|c|c|c|c|}
\hline $\begin{array}{l}\text { S. } \\
\text { no }\end{array}$ & Component & $\begin{array}{l}\text { Score before first cellular } \\
\text { transplantation }\end{array}$ & $\begin{array}{l}\text { Score post first cellular } \\
\text { transplantation }\end{array}$ \\
\hline $\mathbf{E}$ & Sensory aspects & 7 & 6 \\
\hline 5 & Insensitive to pain & 1 & I \\
\hline 6 & $\begin{array}{l}\text { Responds to objects/people unusually by smelling, touching } \\
\text { or tasting }\end{array}$ & 2 & I \\
\hline \multirow[t]{6}{*}{$\mathbf{F}$} & Cognitive component & 12 & 10 \\
\hline & Inconsistent attention and concentration & 2 & I \\
\hline & Shows delay in responding & 3 & 2 \\
\hline & Has unusual memory of some kind & 4 & 4 \\
\hline & Has 'savant' abilities & 5 & 3 \\
\hline & Total & 98 & 81 \\
\hline
\end{tabular}

Table 2 Comparison of pre and post intervention SUV values in 18 FDG PET CT scan of the brain and correlation with clinical symptomatic improvements in the case study

\begin{tabular}{|c|c|c|c|}
\hline Region & $\begin{array}{l}\text { Mean SUV } \\
\text { (Pre) }\end{array}$ & $\begin{array}{l}\text { Mean SUV } \\
\text { (Post) }\end{array}$ & Correlating clinical functional improvements observed in the case \\
\hline Cerebellum Left & 2.67 & 2.8 & \multirow[b]{2}{*}{ Coordination, memory, emotions ,fine motor activity, eye contact } \\
\hline Cerebellum Right & 2.59 & 2.73 & \\
\hline Frontal lobe Left & 4.09 & 3.89 & \multirow{2}{*}{ Initiation, planning, anticipation, organization, problem solving, emotions, attention } \\
\hline Frontal lobe Right & 4 & 3.8 & \\
\hline Parietal lobe Left & 4.08 & 3.94 & \multirow{2}{*}{ Integration of sensory information and language } \\
\hline Parietal lobe Right & 3.98 & 3.83 & \\
\hline Temporal lobe Left & 4.03 & 3.85 & \multirow{2}{*}{ Social interaction, memory and categorizing objects } \\
\hline Temporal lobe Right & 3.9 & 3.75 & \\
\hline
\end{tabular}

\section{Discussion}

Autism is one of the developmental disorders worldwide. The conventional treatments such as Applied Behavioral Analysis (ABA), occupational therapy, speech therapy and pharmacological treatment, mainly aim at managing common symptoms of autism. ${ }^{5}$ These therapies fail to address the core pathology of autism. Hence, establishing a treatment with a potential to reverse the underlying neuropathology of autism is the need of the hour. Recent studies of cellular therapy in children with autism have shown significant clinical improvements. ${ }^{6-13}$

In autism, defect in cortical connectivity and neural migration to cerebral cortex has been observed which may consecutively affect neuronal communication and excitatory-inhibitory balance. ${ }^{14,15}$ Individuals with autism have shown increased levels of pro-inflammatory cytokines along with marked inflammation and microglial activation leading to immune dysregulation. ${ }^{16}$ Inflammatory response is also stimulated by increased oxidative stress due to lack of antioxidants in autism. ${ }^{17}$ Hypoperfusion is also one of the pathophysiologies linked to autism, leading to hypoxia of the brain, causing cell death. ${ }^{18}$ These pathologies affect the brain function and plasticity and could be responsible for the impaired cognition, behaviour, language and communication, social interaction, etc in autism.

Cellular therapy, through its repair and regenerative characteristics may help in regaining lost functions in these individuals. The postulated mechanism of action of cellular therapy involves angiogenesis, neurogenesis, immunomodulation and neuroprotection. We chose autologous BMMNCs for the intervention as they are the most preferred choice of cells due to their safety and feasibility. Their safety and efficacy has already been established in other neurological disorders. ${ }^{19-26}$ These cells are a mixture of various hematopoietic and non hematopoietic cells which are more potent in combination as compared to their individual fractions. On transplantation, they migrate to the damaged areas and initiate repair. These cells are able to differentiate into neuronal cells and replace the lost cells. ${ }^{27}$ They inhibit the pro-inflammatory cytokines and release anti inflammatory molecules which modulates the immune balance and reduce inflammation..$^{28}$ They secrete angiogenic factors and promote angiogenesis thus reversing hypoxia of the brain. ${ }^{29}$ This improves blood supply and oxygenation to the brain. By improving the brain plasticity, cell therapy may help in alleviating the symptoms of individuals with autism.

After intervention, there were marked improvements in cognition, social interaction, behaviour, emotional response and communication of the patient. These improvements were also reflected on the scores of all the outcome measure scales. PET CT scan of the brain which was used as a monitoring tool to study the effect of intervention at a cellular level, also showed improvement in brain metabolism. The areas showing hyper metabolism before the intervention showed reduced metabolic activity after the intervention. Frontal, parietal and temporal lobes showed improvements. The functional improvements 
observed in the patient clinically correlate with the areas showing metabolic changes.

Lack of control is the one of the limitation of this case study. But looking at the significant improvement seen after the cellular transplantation combined with the multidisciplinary rehabilitation, which was not observed previously with only rehabilitation, the patient could serve as a self control.

\section{Conclusion}

This case study suggests that cellular therapy enhances the outcome of standard rehabilitation treatment for autism. This is possible because the cellular therapy repairs the core underlying defect in the brain function. The significant symptomatic improvements are corroborated by improvements in objective scales. PET CT scan of the brain serves as a definitive evidence of improved brain metabolism after the intervention. Larger clinical studies are required of cellular therapy with PET CT scan as a monitoring tool in autism.

\section{Acknowledgements}

None.

\section{Conflict of interest}

The author declares no conflict of interest.

\section{References}

1. Belmonte MK, Allen G, Beckel-Mitchener A, et al. Autism and abnormal development of brain connectivity. J Neurosci. 2004;24(42):92289231.

2. Wegiel J, Kuchna I, Nowicki K, et al. The neuropathology of autism: defects of neurogenesis and neuronal migration, and dysplastic changes. Acta Neuropathol. 2010;119(6):755-770.

3. Park DH, Eve DJ, Chung YG, et al. Regenerative medicine for neurological disorders. Scientific World Journal. 2010;10:470-489.

4. Liu SP, Fu RH, Huang SJ, et al. Stem cell applications in regenerative medicine for neurological disorders. Cell Transplant. 2013;22(4):631637.

5. Myers SM, Johnson CP. Management of children with autism spectrum disorders. Pediatrics. 2007;120(5):1162.

6. Alok S, Nandini G, Hemangi S, et al. Autologous bone marrow mononuclear cell therapy for autism - an open label proof of concept study. Stem cell international. 2013;2013:13.

7. Alok S, Nandini G, Prerna B, et al. An improved case of autism as revealed by PET CT scan of the brain in patient transplanted with autologous bone marrow derived mononuclear cells. J Stem Cell Res Ther. 2013;3:139.

8. Alok S, Nandini G, Akshata S, et al. Autologous bone marrow mononuclear cells may be explored as a novel potential therapeutic option for autism. J Clin Case Rep. 2013;3:282.

9. Alok S, Nandini G, Hemangi S, et al. Intrathecal autologous bone marrow mononuclear cell transplantation in a case of adult autism. Autism open access. 2013;3:113.

10. Alok S, Nandini G, Hemangi S, et al. Cell therapy effects portrayed on positron emission tomography computerized tomography scan of the Stem Cell Therapy In Pediatric Neurological Disorders brain serve as a new dimension for autism: A case report. Journal of Paediatric Neurology. 2014;12:3.
11. Sharma A, Gokulchandran N, Shetty A, et al. Neuropsychiatric disorder tackled by innovative cell therapy-A case report in autism. J Stem Cell Res Transplant. 2014;1(1):4.

12. Alok S, Nandini G, Hemangi S, et al. Amelioration of autism by autologous bone marrow mononuclear cells and neurorehabilitation:A case report. American Journal of Medical Case Reports. 2015;3(10):304-309.

13. Alok S, Nandini G, Hemangi S, et al. PET-CT scan shows decreased severity of Autism after autologous cellular therapy: A case report. Autism Open Access.

14. Minshew NJ, Williams DL. The new neurobiology of autism: cortex, connectivity, and neuronal organization. Arch Neurol. 2007;64(7):945950 .

15. Rubenstein JL, Merzenich MM. Model of autism: increased ratio of excitation/inhibition in key neural systems. Genes Brain Behav. 2003;2(5):255-267.

16. Gottfried C, Bambini-Junior V, Francis F, et al. The impact of neuroimmune alterations in autism spectrum disorder. Front Psychiatry. 2015;6:121.

17. Chauhan A, Chauhan V. Oxidative stress in autism. Pathophysiology. 2006;13(3):171-181.

18. Ichim TE, Solano F, Glenn E, et al. Stem cell therapy for autism. $J$ Transl Med. 2007;5:30.

19. Alok S, Hemangi S, Nandini G, et al. A clinical study of autologous bone marrow mononuclear cells for cerebral palsy patients: a new frontier. Stem Cells International. 2015;2015:11.

20. Alok S, Hemangi S, Pooja K, et al. Cell therapy attempted as a novel approach for chronic traumatic brain injury - a pilot study. Springer Plus. 2015;4:26.

21. Sharma AK, Sane HM, Paranjape AA, et al. The effect of autologous bone marrow mononuclear cell transplantation on the survival duration in Amyotrophic Lateral Sclerosis - a retrospective controlled study. Am J Stem Cells. 2015;4(1):50-65.

22. Sharma A, Sane H, Gokulchandran N, et al. Role of autologous bone marrow mononuclear cells in chronic cervical spinal cord injury-a longterm follow up study. J Neurol Disord. 2013;1:138.

23. Sharma A, Gokulchandran N, Sane H, et al. Detailed analysis of the clinical effects of cell therapy for thoracolumbar spinal cord injury:an original study. Journal of Neurorestoratology. 2013;1:13-22.

24. Sharma A, Sane H, Badhe P, et al. A clinical study shows safety and efficacy of autologous bone marrow mononuclear cell therapy to improve quality of life in muscular dystrophy patients. Cell Transplant. 2013;22(Supp1 1):S127-S138.

25. Alok S, Hemangi S, Nandini G, et al. Autologous Bone Marrow Mononuclear Cells Intrathecal Transplantation in Chronic Stroke Stroke. Research and Treatment. 2014;2014:9.

26. Alok S, Nandini G, Guneet C, et al. Administration of autologous bone marrow derived mononuclear cells in children with incurable neurological disorders and injury is safe and improves their quality of life. Cell Transplantation. 2012;21(Supp 1):S1-S12.

27. Sanchez-Ramos J, Song S, Cardozo-Pelaez F, et al. Adult bone marrow stromal cells differentiate into neural cells in vitro. Exp Neurol. 2000;164(2):247-256.

28. Bifari F, Lisi V, Mimiola E, et al. Immune modulation by mesenchymal stem cells. Transfus Med Hemother. 2008;35(3):194-204.

29. Duffy GP, Ahsan T, O’Brien T, et al. Bone marrow-derived mesenchymal stem cells promote angiogenic processes in a time- and dose-dependent manner in vitro. Tissue Eng Part A. 2009;15(9):2459-2470. 